\title{
The object-based representation of partially occluded surfaces in short-term visual memory: Evidence from image combination
}

\author{
PETER WALKER and ROGER MILES \\ Lancaster University, Lancaster, England
}

\begin{abstract}
Distinct short-term and longer term visual representations of form allow people to combine mental images of separately presented pictures in order to identify a novel object (see, e.g., Brandimonte, Hitch, \& Bishop, 1992; Hitch, Brandimonte, \& Walker, 1995; Walker, Hitch, Dewhurst, Whiteley, \& Brandimonte, 1997). The present study focuses on the short-term representation and asks whether it describes the 2-D features in a picture or the depicted surfaces in 3-D space (see Nakayama, He, \& Shimojo, 1995). To-be-combined figures were sometimes partially occluded by irrelevant forms, and it was determined whether image combination was contingent on the directly visible regions of the figures or on the perceptually completed figures. Results showed that image combination was not impeded by partial occlusion, though it was impeded if an occluder was removed from a picture and only the previously visible regions of the figure were presented. It is concluded that the short-term visual representation supporting image combination is not a pictorial code, but is an object-based description of completed surfaces in 3-D space.
\end{abstract}

In previous studies, an image combination task has been used to investigate the visual representation of form (Brandimonte, Hitch, \& Bishop, 1992; Hitch, Brandimonte, \& Walker, 1995; Walker, Hitch, Dewhurst, Whiteley, \& Brandimonte, 1997). In this task, subjects combine mental images of two successively presented pictures in order to identify an emergent form. The emergent form cannot be identified from either of the component images alone; instead, it requires the images to be accurately superimposed. In a short-term memory condition, the to-be-combined visual stimuli are presented in immediate succession. In a long-term memory condition, there is a learning phase during which the set of first members of the pairs of tobe-combined stimuli are repeatedly presented until the subject can visualize each one in turn. Then, in the testing phase, the second member of each pair is presented in turn and subjects combine each one with an image of its partner from the learned set.

Two distinct types of visual representation of form have been found to support image combination (Brandimonte et al., 1992; Hitch et al., 1995; Walker et al., 1997). The first is a detailed description of a pictured object that preserves information about surface features (e.g., color). The second is a more abstract, structural description that excludes surface information but is still sufficiently close to the picture (with regard to details of shape, position, and size of the figure) that it can reveal the emergent form. ${ }^{1}$

The authors are grateful to Graham Hitch and Helen Whiteley for advice at various stages of this research. Correspondence concerning this article should be addressed to P. Walker, Department of Psychology, Lancaster University, Lancaster LAl 4YF, England (e-mail: p.walker@lancaster.ac.uk).
It appears that the first type of representation supports image combination when either of two conditions holdsthat is, when verbal recoding of the picture is precluded, or when the procedure requires short-term rather than longterm retention of the first to-be-combined image. Under these conditions, people find it more difficult to combine the images of two pictures when they are incongruent in color (i.e., when a black-on-white line drawing has to be combined with a white-on-black drawing), confirming that this type of representation preserves surface information. However, when verbal recoding is permitted and longer term retention of the first image is required, the structural description supports image combination and the color congruity of the to-be-combined pictures no longer affects performance (Hitch et al., 1995).

Walker et al. (1997) elaborate further on the nature of the short-term representation. They represented the figures in the to-be-combined pictures as solid forms, to allow color congruity to be varied independently for figure and ground. The results showed a clear impairment in image combination when the to-be-combined figures were incongruent in color (black on gray and white on gray) but not when their grounds were incongruently colored (gray on black and gray on white). This sensitivity to the color congruity of the to-be-combined figures was observed only in association with the short-term representation. In this way, this representation appears to be objectbased and distinct both from the structural representation and from a 2-D representation of the whole picture (i.e., it is not the pictorial code of Bruce \&.Young, 1986). Using more conventional memory tasks, Wilton (1989) and Walker and Cuthbert (1998) have also provided evidence for an object-based visual representation that preserves 
information about both the form and color of a pictured object.

The present study focuses on the short-term representation supporting image combination. We ask whether this representational form is based on a description of the 2-D features in a picture or on a description of the depicted surfaces in 3-D space. Nakayama, He, and Shimojo (1995) have reviewed recent evidence contrasting the functional significance of these two levels of description. Because it is assumed that surface representations take account of partial occlusion, a common research strategy has been to determine whether it is the characteristics of the directly visible regions of a partially occluded surface that support a perceptual phenomenon, or the characteristics of the completed surface. According to Nakayama et al., despite being the product of sophisticated processes that take partial occlusion into account, surface representations are derived quickly and fluently and can support low-level perceptual phenomena (such as visual search, movement perception, object segregation, and object identification).

In the present study, a short-term memory procedure is combined with articulatory suppression to ensure that image combination relies on the targeted representational form, and the to-be-combined figures are some- times partially occluded by an irrelevant form. The main aim is to determine whether image combination operates on representations of completed surfaces, rather than on representations of the directly visible regions of figures. A subsidiary aim is to determine whether image combination is equally effective when to-be-combined figures are partially occluded and when they are not.

The to-be-combined pictures, based on those used by Hitch et al. (1995) and Walker et al. (1997), depicted objects as solid shapes rather than as the line drawings originally created by Brandimonte et al. (1992). There were six pairs of pictures, with each pair, when combined, representing a familiar object. The picture pairs are illustrated in Figure 1, along with a complete version of each emergent object, in order to illustrate how these objects would be depicted if the pictures themselves were combined according to the imagery instructions.

In addition to a complete version (complete) of the first picture in each pair, two further versions were prepared. In one of these (holes), a black mask with holes punched in it was added to the picture. The mask partially occluded the original figure, creating the impression of an object being viewed through, and continuing beyond, the holes in a solid black surface. A different mask was created for each picture. In another version (fragments),
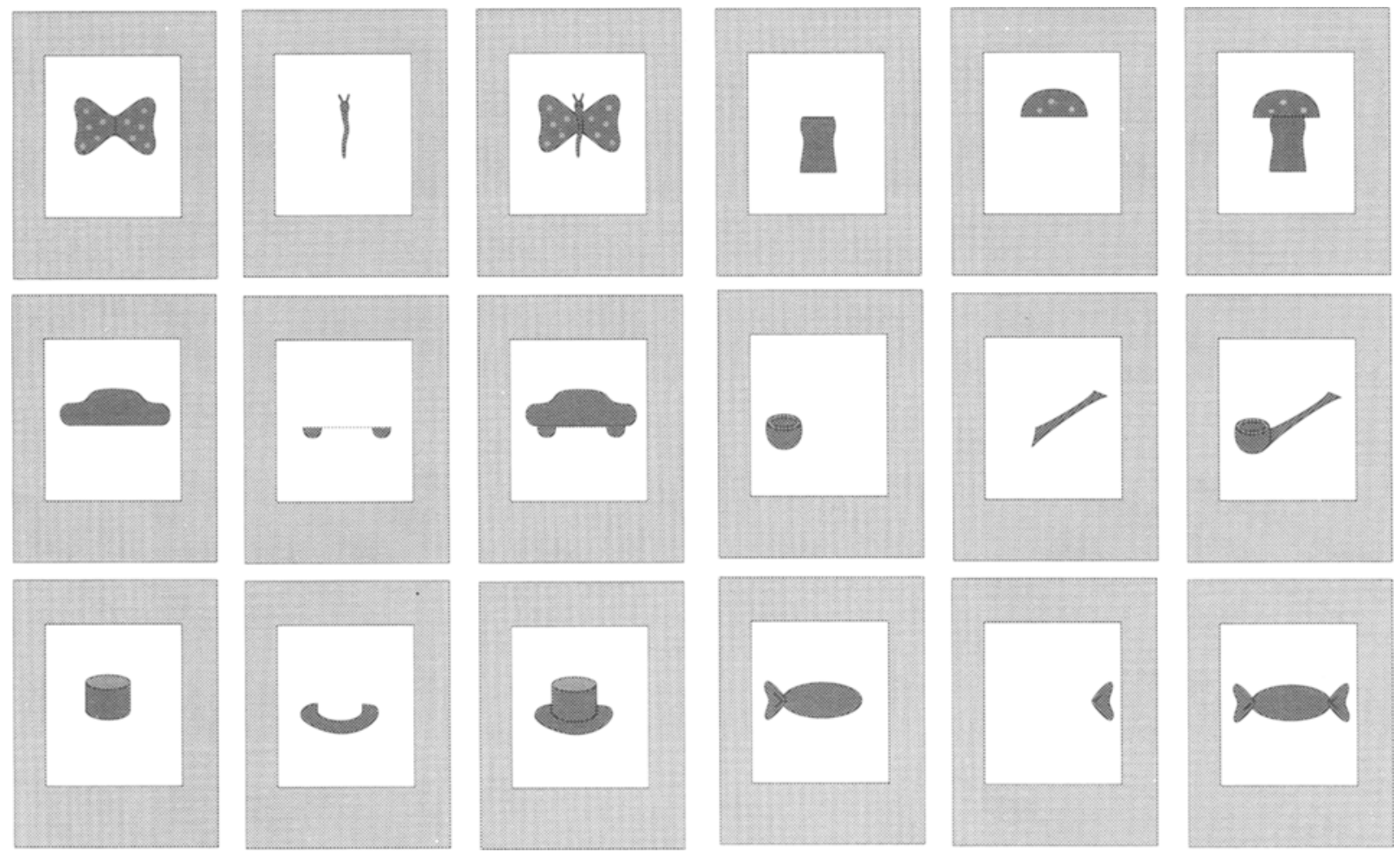
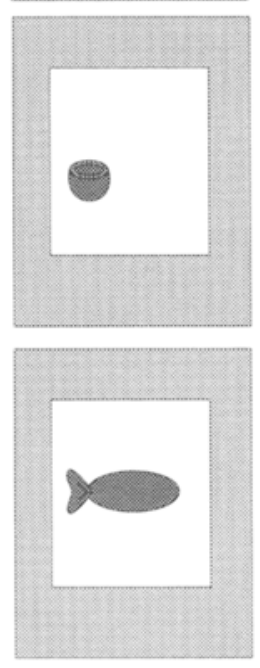
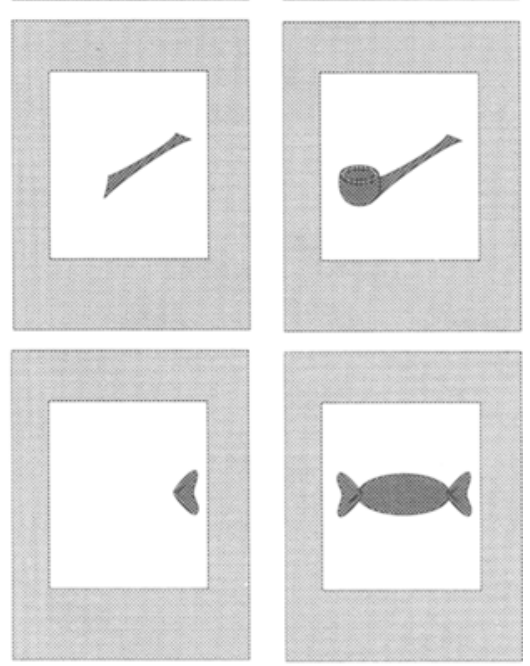

Figure 1. The six picture pairs used in the image combination task, together with combined versions illustrating how the emergent objects would be depicted if the pictures themselves were combined according to the imagery instructions. Though illustrated here and in Figure 2 as gray figures, for the experiment itself the object parts were printed in red ink to aid their segregation from the black, occluding surfaces. 
there was no mask, yet only the regions of the figure that were directly visible through the holes in the masked version were presented.

In addition to a complete version (complete) of the second picture in each pair, a further version was prepared. In this version (blobs), solid black shapes were added to partially occlude the original figure. These occluding blobs exactly matched, both in shape and location, the holes in the mask created for the picture with which it was paired. These different versions are illustrated in Figure 2 with reference to the picture of a butterfly.

The style of the occluders was inspired by Bregman's (1981) illustration of some of the principles underlying amodal completion. As Nakayama et al. (1995) have explained, three fundamental principles are associated with the amodal completion of partially occluded surfaces. First, only one of two adjoining regions is perceived to own their shared border. Second, ownership of the border is associated with a region's being perceived to be closer to the viewer (i.e., the occluder owns the shared border). Third, a region that does not own a particular border is unbounded and may connect with other partially occluded and unbounded regions to form a larger surface continuing behind the occluder. These principles offer a way of understanding why the figures in the six pairs of pictures would undergo amodal completion when partially occluded. Equally, the same principles explain why, when an occluder is removed, the fragments that remain are less likely to be completed. The borders of the fragments that were originally shared with the occluder can no longer be attributed to an occluder that is physically present, but instead will tend to be perceived to be owned by the fragments themselves. As a result, the fragments have no unbounded regions by which they can connect with each other.

Many of the visual cues supporting amodal completion have now been identified and could be employed directly and formally to design an occluder (see Kellman \& Shipley, 1991, for a thorough treatment of the perception of partly occluded objects). In the present case, however, the occluders were designed intuitively to satisfy a number of requirements. With regard to the first member of each pair of to-be-combined figures, the holes in the occluding surface needed to permit completion, allowing the figure to be correctly identified. To facilitate this, the occluder and occluded surfaces formed $T$ junctions. In addition, however, there was the requirement that, when an occluder was removed, the figure fragments that remained would be less likely to be completed, or to be grouped in a way that permitted identification of the figure. Finally, there was the simultaneous requirement that when an occluder with holes was replaced by the corre-
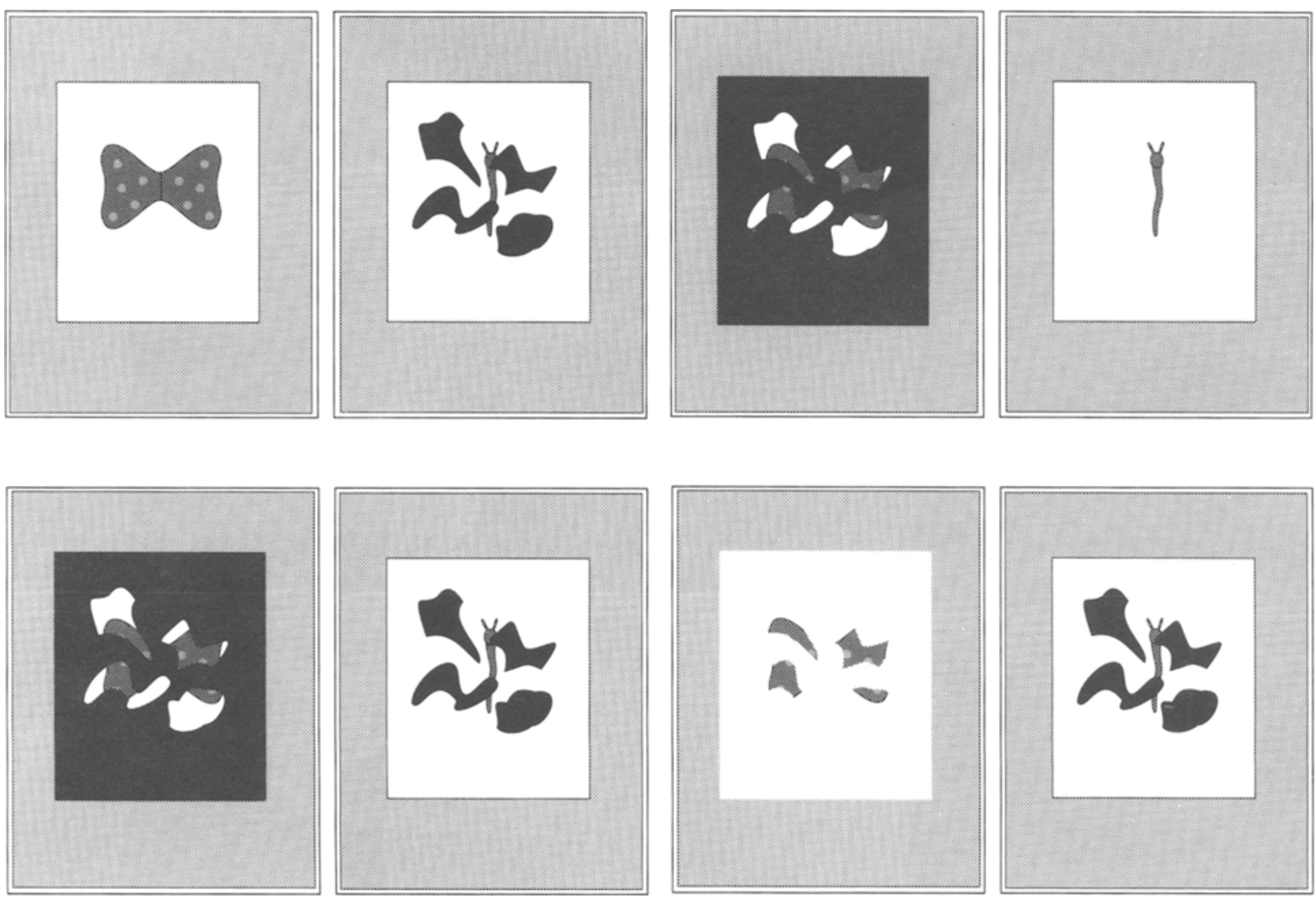

Figure 2. Different versions of the to-be-combined pictures depicting the butterfly. 
sponding blobs, these would allow completion and identification of the second member of each pair of to-becombined figures.

The different versions of the first- and second-presented pictures were combined in the six ways possible: completecomplete, holes-complete, fragments-complete, completeblobs, holes-blobs, fragments-blobs. If image combination is not restricted to operating on directly visible regions, but instead can operate on amodally completed surfaces, then, to the extent that completion does not occur with the fragments versions of the pictures, performance should be significantly worse with these stimuli than with the holes versions of the pictures. Furthermore, if image combination can operate on amodally completed surface representations, unperturbed by partial occlusion, then comparable levels of performance should be observed in all conditions except those involving the fragments versions of the first figures. That is, provided viewing times are sufficient to allow amodal completion to occur, then equivalent levels of performance should be observed for the complete and holes versions of the first pictures, and for the complete and blobs versions of the second pictures. Indeed, if image combination is based on representations of completed figures that are separate from any representation of the occluders, then performance in the holesblobs condition should be comparable to that in other conditions, despite the fact that a literal combination of the exactly complementary occluders would yield an occluder covering the entire picture region. In summary, if image combination operates on completed surfaces, rather than on the directly visible regions of the figures, and does so equally effectively whether or not the figures are partially occluded, then performance should be relatively poor in the two fragments conditions, but should otherwise be equivalent across the six conditions.

\section{METHOD}

\section{Subjects \\ One hundred fourteen volunteers in the age range 18-52 took part in the main study. They were all students at Lancaster Univer- sity who were tested individually in their hall of residence or in the first author's office.}

\footnotetext{
Materials

The pictures were prepared in Macromedia Freehand (Version 5.5) on a Power Macintosh 8500/120 and were printed on a QMS Magicolor laser printer. The six objects used in previous studies were again used here, with one exception: The picture of a skipping rope was not amenable to manipulations involving partial occlusion and was replaced by a picture depicting a top hat. The six objects were a butterfly, motor car, top hat, mushroom, smoker's pipe, and toffee. An additional pair of pictures depicting the container and handle of a drinking mug were prepared on transparencies and were used to explain the requirements of the task to the subjects.

Each picture was printed on a $17 \times 22 \mathrm{~cm}$ laminated sheet and incorporated a medium gray border framing a central white area measuring $11 \times 14 \mathrm{~cm}$. Within this central area, the object part was printed in red (Munsell coordinates: hue $=5 \mathrm{R}$, value $=4$, and chroma $=14$ ) with a fine black outline. Occasionally, a slightly lighter red tint (hue $=5 \mathrm{R}$, value $=5$, and chroma $=10$ ) was added
}

to aid identification of an object (as with the spots on the butterfly wings, for example). The relative dimensions of the various figures were as in Figure 1.

\section{Design}

Nineteen subjects were assigned to each of the six experimental conditions established by selecting different versions of the pictures for image combination. The assignment of subjects was random, except for the constraint that the six groups had the same gender ratio ( 10 females, 9 males). A random order of presentation for the six objects was determined for each of the 19 subjects, and the same set of orderings was then used for the subjects in all six conditions.

\section{Procedure}

Prior to the experimental trials, the transparencies were used to give instructions to the subjects regarding the image combination task. The transparency of the container of the drinking mug was presented for $3 \mathrm{sec}$, followed by presentation of the handle for $3 \mathrm{sec}$. The experimenter then slid one transparency over the other to demonstrate how, when the picture borders were aligned, superimposition of the two patterns created a novel figure. The subject was asked to name the figure resulting from the combination. Additional transparencies of the drinking mug were produced to illustrate the particular nature of the task in the conditions involving the blobs, holes, and fragments versions of the pictures. With the first two of these versions, it was explained that the to-be-combined figures were depicted to give the impression that they were located behind, and partially hidden by, either irregular black shapes or a black screen with irregular holes in it. It was further explained that these black forms were incidental to the task of identifying the objects that would emerge when the red figures were combined. For the subjects being exposed to fragmented pictures, it was explained that the first of each pair of to-be-combined figures would have irregularly chosen regions removed, leaving unconnected figure fragments in their original positions. The subjects were encouraged to identify the original figure, despite the missing information, in order to combine it with the second-presented figure.

Each subject was then shown the card containing the first member of the first pair of experimental stimuli for $3 \mathrm{sec}$. The card was removed from view and replaced by the second member of the pair, again for $3 \mathrm{sec}$. This was then covered by a blank white card and the subject was asked to combine the two stimuli in his/her mind's eye and to identify the new figure resulting from the combination. This procedure was repeated for all six pairs of experimental stimuli. Starting just prior to the presentation of a picture pair, and continuing until after the second member had been hidden from view, the subjects were required to repeatedly and continuously utter "la, la, la,...," at a level that was just audible to the experimenter.

\section{RESULTS}

\section{Supplementary Observations}

Before we report the main image combination results, it is instructive to consider supplementary evidence provided by an additional, though equivalent, group of $50 \mathrm{stu}-$ dents at Lancaster University (age range, 18-27). This evidence concerns the probability with which a correct object identification response could be based on elements of the situation other than a properly combined image. The image combination task was explained to these subjects, using the transparencies, in the same way as for the main group of subjects. Then, one group of 25 subjects was presented with the complete version of the first member of each picture pair and asked to guess what object would be revealed 
by adding another, unspecified part. The mean probability with which the final object was guessed on this basis was only $4.0 \%$. Another group of 25 subjects was presented with a complete version of the second member of each picture pair and asked to guess what object would be revealed by adding another, unspecified part. The mean probability with which the final object was correctly identified on this basis was higher, at $25 \%$, with half of the correct responses being associated with the mushroom. This value represents a baseline against which the success of image combination might best be judged, and with this in mind, we have incorporated it in Figure 3. Finally, the subjects in both groups were presented with complete pictures of the six objects (see Figure 1), and $98 \%$ of their responses correctly identified the objects.

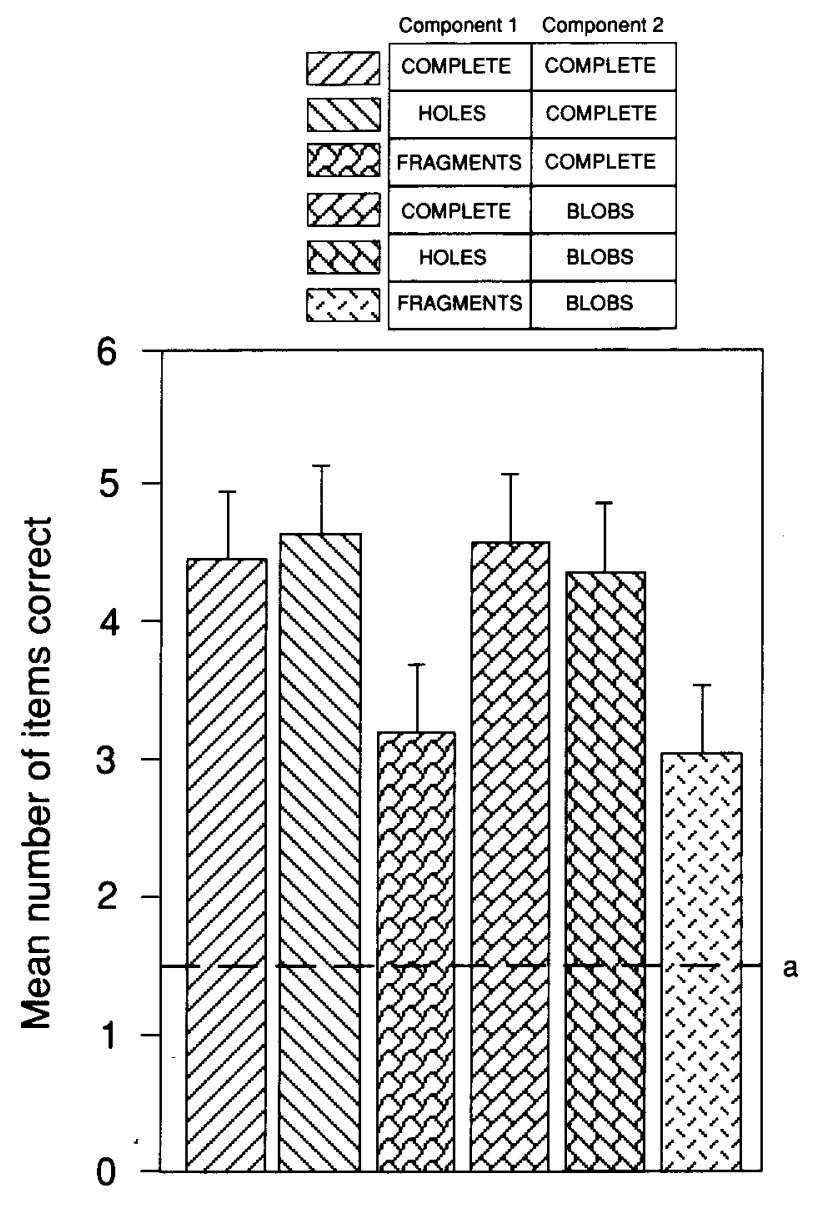

\section{Condition}

Figure 3. The mean number of correct image-combination responses to the set of six objects (with the confidence interval appropriate for evaluating specific differences in performance across conditions). The level of performance indicated by line $a$ corresponds to the probability with which an object could be correctly identified on the basis of viewing just a complete version of the second to-be-combined picture. This provides a baseline against which the success of image combination can be judged.

\section{Image Combination}

The most frequent identification responses in the combination task coincided with the responses given by the supplementary group of subjects, again confirming the success with which the intended objects had been depicted in the pictures. Other responses were counted as correct if they were an obvious synonym of these. For each of the six conditions, Figure 3 shows the mean number of correct image combination responses to the set of six objects, along with the confidence interval appropriate for testing the significance of differences among the conditions (as recommended by Loftus \& Masson, 1994). It is clear from inspection that image combination was significantly poorer, and equally so, in the fragments-complete and fragments-blobs conditions, but was otherwise constant across conditions. This was confirmed by analysis of variance with condition as a between-subjects factor. The main effect of condition was significant $[F(5,108)=$ $8.44, p<.01]$, and a planned comparison confirmed that performance in the fragments-complete and fragmentsblobs conditions was significantly worse than performance in the other four conditions $[F(1,108)=41.34, p<.01]$. This contrast accounted for $98 \%$ of the condition variance.

The overall level of successful image combination varied across the six items, with the mean number of subjects (out of 19) combining each item correctly (across conditions) being $14.8,15.0,10.5,17.5,10.0$, and 9.2 , for the butterfly, motor car, top hat, mushroom, smoker's pipe, and toffee, respectively. An items-based analysis of variance, dealing with the number of subjects correctly combining a picture pair, yielded a pattern of significant results across conditions that was identical to the one observed in the subjects-based analysis. The mean number of subjects correctly combining a pair of figures (with standard deviations in parentheses) was $14.17(4.31), 14.67(2.42)$, $10.17(2.40), 14.50$ (4.68), 13.83 (3.06), and 9.67 (5.46), for the complete-complete, holes-complete, fragmentscomplete, complete-blobs, holes-blobs, and fragmentsblobs conditions, respectively. The main effect of condition was significant $[F(5,25)=6.96, p<.01]$, and the confidence interval appropriate for testing the significance of differences among the conditions (see above) was \pm 1.78 . A planned comparison confirmed that performance in the fragments-complete and fragments-blobs conditions was significantly worse than performance in the other four conditions $[F(1,25)=34.08, p<.01]$. This contrast, which accounted for $98 \%$ of the condition variance, illustrates that the relatively poor performance associated with the fragments versions of the pictures was consistent across the six items. This is also apparent from inspection of the relevant means. Collapsed across the fragmentscomplete and fragments-blobs conditions, the mean number of subjects combining an item correctly was $13,10.5$, $9,15,7$, and 5 , for the butterfly, motor car, top hat, mushroom, smoker's pipe, and toffee, respectively. Collapsing across the other four conditions, the corresponding val- 
ues were consistently higher at 15.75, 17.25, 11.25, 18.75, 11.5 , and 11.25 , respectively.

\section{DISCUSSION}

The pattern of performance across the six conditions indicates that the short-term visual representation supporting image combination incorporates a description of depicted surfaces in 3-D space, rather than a description of 2-D, pictorial features. Support for this comes from at least three aspects of the data. First, the equivalent levels of performance across the complete-complete and holescomplete conditions and across the complete-complete and complete-blobs conditions confirms that image combination can operate on amodally completed representations, unperturbed by the partial occlusion of either tobe-combined figure. Second, the significantly poorer performance in the fragments-complete and fragmentsblobs conditions in comparison with the holes-complete and holes-blobs conditions confirms that image combination does not operate on a representation that is restricted to the directly visible regions of the first to-becombined figure, but instead operates on an amodally completed representation. Third, the holes-blobs condition yielded performance levels equivalent to those observed in both the holes-complete and complete-blobs conditions, indicating that the amodally completed representations of the figures was separate from representations of the occluders. Furthermore, because the occluding forms that were paired together in the holes-blobs condition were exactly complementary to each other, image combination appears not to be the functional equivalent of fusing two pictorial representations, as if one were superimposing two transparencies on an overhead projector. Were this the case, the two occluding black surfaces would have completely filled the area in which the object was depicted and would have had a very disruptive effect on its identification. Thus, the results from the holes-blobs condition provide converging evidence for the separate representation of occluded and occluding figures, and, more generally, for the objectbased nature of the short-term visual representation supporting image combination (see Walker et al., 1997). Together, these various comparisons confirm that image combination operates on amodally completed surface representations and, at least under the conditions of the present study, does so just as effectively as when the figures are completely visible.

Performance in the fragments-complete condition was better than would have been expected given the success with which the supplementary group of subjects had been able to identify the emergent objects on the basis of the complete version of the second to-be-combined stimulus. This might have arisen from the first-presented figure being identified, on some occasions, on the basis of visual features remaining in the fragments. Alternatively, memory of where the fragments were located, when considered along with the second-presented stimulus, might have constrained the possibilities regarding what the combined object could be. For our present purposes, however, the important observation is that performance was significantly poorer with the fragmented version of each picture.

In summary, the present study has demonstrated that the short-term visual representation supporting image combination is not a representation of the 2-D features in a picture- that is, it is not a pictorial representation. Instead, in addition to preserving information about figure separately from ground (see Walker et al., 1997, and Walker \& Cuthbert, 1998), it is a representation that incorporates descriptions of completed surfaces in 3-D space and that segregates information about surfaces belonging to different objects.

It remains uncertain whether, in the present study, amodal completion ever took place after the pictures had been removed from view and, therefore, were available only in short-term memory. According to Kosslyn (1994), one function of the visual buffer is to allow representations to be transformed. ${ }^{2}$ Although there is evidence that novel interpretations of pictures can emerge (be discovered) from a memory representation (Brandimonte \& Gerbino, 1993; Finke, Pinker, \& Farah, 1989), the authors are unaware of any demonstration that amodal completion can occur after a picture has been removed. But, in any case, a value of $3 \mathrm{sec}$ for the exposure duration of each to-be-combined picture was considered long enough for amodal completion to occur for the holes and blobs pictures. Our observations of subjects in the present study incline us to believe that amodal completion did take place while the pictures remained in view, and this is reinforced by other evidence that memory representations of amodally completed surfaces can be established relatively quickly.

Sekuler and Palmer (1992) investigated how performance in a shape matching task was facilitated by the prior presentation of one of the shapes, which, on different occasions, appeared complete, partially occluded, or in a fragmented form corresponding to the regions not hidden by the occluder. With very brief presentation of a prime shape $(<200 \mathrm{msec})$, they found that priming was based on the visible 2-D features of the shape, with a partially occluded prime behaving like the corresponding fragmented prime. But when presented for longer periods, a partially occluded prime behaved like a complete prime, implying that priming was based on an amodally completed representation of the shape. Further evidence that view-independent memory representations can be established relatively quickly has been provided by Epstein and his colleagues (Epstein \& Broota, 1986; Epstein, Hatfield, \& Muise, 1977; Epstein \& Lovitts, 1985). They studied explicit recognition memory for shapes cut out of posterboard (each one presented for just $1 \mathrm{sec}$ ) and contrasted the contribution of preconstancy representations of shape and size, which reflect the properties of the 2-D proximal stimulus, against the contribution of postconstancy, object-based representations. Provided that subjects attended to the shapes at presentation, explicit recognition memory was based on postconstancy repre- 
sentations. Only when subjects did not attend to the shapes, despite looking at them, were recognition decisions based on preconstancy representations.

In agreement with the results of the present study, Hock and Schmelzkopf (1980) have demonstrated that visual memory for a photographed real-world scene need not be restricted to the visual details explicitly contained in the picture. Rather, their subjects derived a schematic, visuospatial representation of the 3-D layout of the scene and the objects in it. This representation was sufficiently viewpoint independent to allow the scene to be recognized just as well from a new photograph, taken from a different location, as from the original photograph. If it is assumed that the specific nature of any partial occlusion of objects in the original photograph would not be reproduced in a new photograph, this result implies that occlusion was taken into account, and that representations of completed objects established.

In a different way, Helene Intraub and her colleagues (e.g., Intraub \& Bodamer, 1993; Intraub, Gottesman, Willey, \& Zuk, 1996) have also demonstrated that visual memory for a picture need not be a low-level, sensory representation of the details explicitly contained in the picture, but can be a description of what is perceived to be the more complete nature of the scene. They found that people can derive an abstract, schematic visual representation of the spatial and form-related aspects of a scene that incorporates information about elements lying just outside the picture. It is as if the picture border is treated just as the occluding forms in the present image combination study, and people remember what they perceived to be the more complete nature of the scene. Thus, the schematic representation of a scene can go beyond what is directly available in the pictured field of view, giving rise to a form of completion referred to as boundary extension. In relation to the possible time course of the events under investigation in the present study, it is worth noting that Intraub et al. observed that the derivation of the schematic visual representation occurred as a direct result of perceptual processing, with picture presentation times of 250-350 msec.

Intraub et al. (1996) compare the schematic visual representation giving rise to boundary extension with the type of representation underlying the integration of visual information from successive glimpses of a scene. They point to a growing body of evidence indicating that such integration is based on a schematic, nonsensory visuospatial representation (see Hochberg, 1986; Irwin, 1991; Rayner \& Pollatsek, 1992). For example, Irwin (1991) has demonstrated that the integration of visual information across successive eye fixations does not reflect the fusion of two sensory representations fixed in spatiotopic coordinates. Rather, he shows that the representations resemble the short-term visual memory that Phillips (1974) had earlier contrasted with sensory visual memory. These representations were found to be less detailed, more schematic, and longer lasting than sensory visual memory, and to be framed in object-based coordinates rather than viewer-centered or environment-centered coordinates. Interestingly, Irwin concludes that the integration of information from successive eye fixations does not reflect processes "analogous to the overlaying of transparencies on an overhead projector" (p. 421). It has already been commented that the present results, especially those from the holes-blobs condition, indicate that image combination also is not analoguous to the overlaying of transparencies, despite the way in which transparencies were used to explain the requirements of the task to the subjects! It appears that image combination, though contrived, may well provide additional and converging evidence about how a general purpose, short-term visual memory is able to support visual integration in more naturalistic situations.

\section{REFERENCES}

Brandimonte, M. A., \& Gerbino, W. (1993). Mental image reversal and verbal recoding: When ducks become rabbits. Memory \& Cognition, 21, 23-33.

Brandimonte, M. A., Hitch, G. J., \& Bishop, D. V. M. (1992). Manipulation of visual mental images in children and adults. Journal of Experimental Child Psychology, 53, 300-312.

Bregman, A. S. (1981). Asking the "What for" question in auditory perception. In M. Kubovy \& J. R. Pomerantz (Eds.), Perceptual organization (pp. 99-118). Hillsdale, NJ: Erlbaum.

Bruce, V., \& YounG, A. W. (1986). Understanding face recognition. British Journal of Psychology, 77, 305-327.

EPSTEIN, W., \& BRoOTA, K. D. (1986). Automatic and attentional components in perception of size-at-a-distance. Perception \& Psychophysics, 40, 256-262.

EPSTEIN, W., Hatfield, G., \& Muise, G. (1977). Perceived shape at a slant as a function of processing time and processing load. Journal of Experimental Psychology: Human Perception \& Performance, 3, 473-483.

EPSTEIN, W., \& LoviTTS, B. E. (1985). Automatic and attentional components in perception of shape-at-a-slant. Journal of Experimental Psychology: Human Perception \& Performance, 11, 355-366.

FinKe, R. A., Pinker, S., \& Farah, M. J. (1989). Reinterpreting visual patterns in mental imagery. Cognitive Science, 13, 51-78.

Hitch, G. J., Brandimonte, M. A., \& Walker, P. (1995). Two types of representation in visual memory: Evidence from the effects of stimulus contrast on image combination. Memory \& Cognition, 23, 147-154.

HochBERG, J. (1986). Representation of motion and space in video and cinematic displays. In K. R. Boff, L. Kaufman, \& J. P. Thomas (Eds.), Handbook of perception and human performance (Vol. 1, pp. 22.122.64) New York: Wiley.

Hock, H. S., \& SchMELzKOPF, K. F. (1980). The abstraction of schematic representations from photographs of real-world scenes. Memory \& Cognition, 8, 543-554.

InTRAuB, H., \& BoDAmer, J. L. (1993). Boundary extension: Fundamental aspect of pictorial representations or encoding artifact? Journal of Experimental Psychology: Learning, Memory, \& Cognition, 19, $1387-1397$.

Intraub, H., Gottesman, C. V., Willey, E. V., \& ZUK, I. J. (1996). Boundary extension for briefly glimpsed photographs: Do common perceptual processes result in unexpected memory distortions? Journal of Memory \& Language, 35, $118-134$.

IRWIN, D. E. (1991). Information integration across saccadic eye movements. Cognitive Psychology, 23, 420-456.

Kellman, P. J., \& Shipley, T. F. (1991). A theory of visual interpolation in object perception. Cognitive Psychology, 23, 141-221.

KossLyn, S. M. (1994). Image and brain. Cambridge, MA: MIT Press. LofTus, G. R., \& MASSON, M. E. J. (1994). Using confidence intervals in within-subject designs. Psychonomic Bulletin \& Review, 1, 476-490.

Nakayama, K., He, Z. J., \& Shimojo, S. (1995). Visual surface representation: A critical link between lower-level and higher-level vision. In S. M. Kosslyn \& D. N. Osherson (Eds.), Visual cognition: An invitation to cognitive science (Vol. 2, pp. 1-70). Cambridge, MA: MIT Press. 
PhiLlips, W. A. (1974). On the distinction between sensory storage and short-term visual memory. Perception \& Psychophysics, 16, 283-290.

Rayner, K., \& Pollatsek, A. (1992). Eye movements and scene perception. Canadian Journal of Psychology, 46, 342-376.

SeKuler, A. B., \& Palmer, S. E. (1992). Perception of partly occluded objects: A microgenetic analysis. Journal of Experimental Psychology: General, 121, 95-111.

WALKER, P., \& CuthBert, L. (1998). Remembering visual feature conjunctions: Visual memory for shape-color associations is object-based. Visual Cognition, 5, 409-455.

Walker, P., Hitch, G. J., Dewhurst, S. A., Whiteley, H. E., \& BRANDIMONTE, M. A. (1997). The representation of nonstructural information in visual memory: Evidence from image combination. Memory \& Cognition, 25, 484-491.

WILTON, R. N. (1989). The structure of memory: Evidence concerning the recall of surface and background color of shapes. Quarterly Journal of Experimental Psychology, 41A, 579-598.

\section{NOTES}

1. In the present context, the abstractness of a representation reflects the degree to which it preserves information about a scene in a way that is view independent. Postconstancy representations of the shapes and sizes of objects are, in this sense, more abstract than preconstancy representations. Put more generally, descriptions framed in environmentbased or object-based coordinates are more abstract than descriptions framed in viewer-based coordinates. Whereas shape constancy permits a shape to be identified despite variations in its orientation relative to the viewer, size constancy permits an object to be identified despite changes in viewing distance. Amodal completion might be viewed as the product of an equivalent "taking into account" process, which permits a partially occluded object to be identified despite changes in the angle from which it, and its occluder, are viewed. Thus, in the same way as constancy mechanisms culminate in an object-based representation of the shape and size of an object, amodal completion might reflect processes culminating in an object-based representation that is independent of how the object is occluded when viewed from a particular position.

2 . We are grateful to an anonymous reviewer for drawing our attention to the implications of Kosslyn's (1994) thesis for the issues under investigation.

(Manuscript received October 6, 1997; revision accepted for publication May 26,1998 .) 